\title{
Design and implementation of a hospital information system for the Palestine Red Crescent Society in Lebanon
}

L. Rossi, ${ }^{1}$ E. Materia, ${ }^{1}$ A. Hourani, ${ }^{2}$ H. Yousef,${ }^{3}$ V. Racalbuto, ${ }^{4}$ C. Venier ${ }^{4}$ and M. Osman ${ }^{2}$

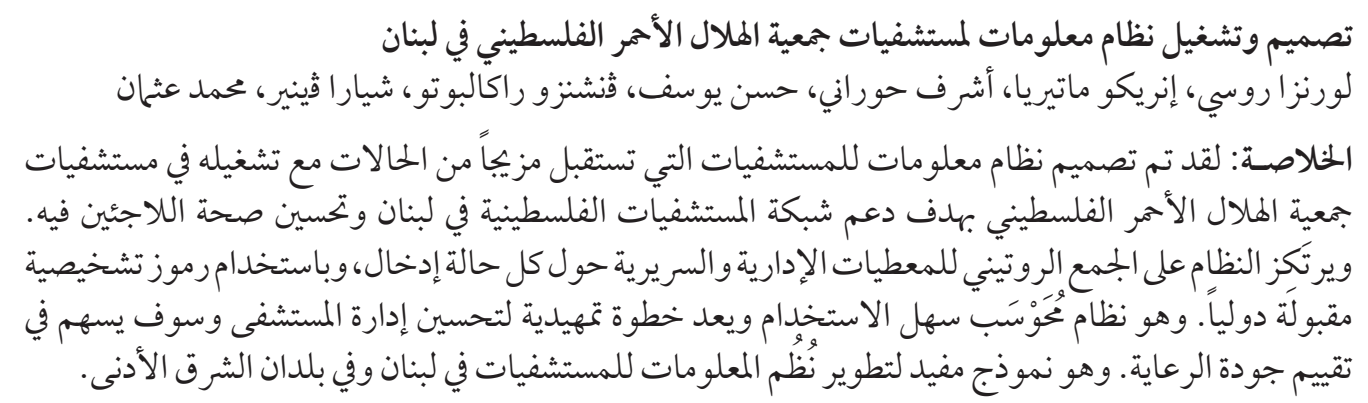

ABSTRACT A case-mix hospital information system was designed and implemented in Palestine Red Crescent Society hospitals in order to support the network of Palestinian hospitals in Lebanon and to improve the health of refugees in the country. The system is based on routine collection of essential administrative and clinical data for each episode of hospitalization, relying on internationally accepted diagnostic codes. It is a computerized, user-friendly information system that is a stepping-stone towards better hospital management and evaluation of quality of care. It is also a useful model for the development of hospital information systems in Lebanon and in the Near East.

\begin{abstract}
Conception et mise en œuvre d'un système d'information hospitalier destiné au CroissantRouge palestinien au Liban

RÉSUMÉ Un système d'information hospitalier de type " case-mix » a été conçu et mis en place dans les hôpitaux du Croissant-Rouge palestinien afin d'aider le réseau des hôpitaux palestiniens au Liban et d'améliorer la santé des réfugiés dans ce pays. Ce système repose sur la collecte systématique de données administratives et cliniques essentielles correspondant à chaque épisode d'hospitalisation, en fonction de codes de diagnostic reconnus à l'échelle internationale. II s'agit d'un système informatisé convivial qui va permettre d'améliorer sensiblement la gestion des hôpitaux et l'évaluation de la qualité des soins. Ce système constitue également un modèle utile pour la mise au point de systèmes d'information hospitaliers au Liban et au Proche-Orient.
\end{abstract}

${ }^{1}$ Agency for Public Health, Lazio Region, Rome, Italy (Correspondence to L. Rossi: rossiquick@gmail.com; Irossi@unicef.org).

${ }^{2}$ Palestine Red Crescent Society, Lebanon Branch, Beirut, Lebanon.

${ }^{3}$ Independent Consultant, Lebanon.

${ }^{4}$ General Directorate for Cooperation for Development, Ministry of Foreign Affairs, Rome, Italy.

Received: 15/06/06; accepted: 27/11/06 


\section{Introduction}

Approximately 400000 Palestinian refugees currently live in Lebanon [1], displaced since the diasporas that followed the 1947 Arab-Israeli war, and the 1967 6-day war. Around $53 \%$ of registered Palestinian refugees still reside in 12 refugee camps scattered throughout the country, suffering critical problems with housing, water supply and sanitation [1]. A high rate of unemployment [2] binds their survival to a subsistence economy, dependant mainly on foreign remittances and international assistance.

Despite these difficulties, regional development and international aid have helped the refugee population in Lebanon reach a stage in the demographic and epidemiological transition similar to that of the host country $[1,3]$. Much of the support for the registered Palestinian population in Lebanon has been secured by the United Nations Relief and Works Agency for Palestine Refugees in the Near East (UNRWA). Assistance to the refugee population is also provided by the Palestine Red Crescent Society (PRCS), whose Lebanon branch operates 6 hospitals in the country, as well as other health and social services.

In 1998, the working group on Palestinian refugees within the multilateral peace negotiations in the Middle East assessed the hospital care needs of Palestinian refugees, and advised that the existing gaps in care were a key humanitarian issue deserving urgent attention. As a result, UNRWA contracted renovated hospitals from PRCS, shifting bed-days from Lebanese private hospitals to a friendlier environment for refugees, and donors pledged to improve PRCS hospital performance. One issue raised by the international community was the need for health data to monitor the health status of Palestinian refugees and the health service activities provided.
Around much of the world, health information systems have been set up to provide routine data in support of a wide range of functions to improve quality of care. Service delivery, disease control, financial control, human and material resource management and the best allocation of limited resources, policy formulation, epidemiological analysis and performance profiling, all need the timely use of good quality data for effective planning and operation. More countries are now introducing standard nomenclature and classification systems, and organizing workshops on health statistics, data analysis and information use for programme managers and health planners. In the Near East as well, several national initiatives aimed at strengthening hospital information systems are in progress, such as those in Jordan [4,5], Syrian Arab Republic and Lebanon itself [6 and Najjar F, unpublished document].

In this paper we report on the process and achievements of a project designed to develop a case-mix hospital information system in PRCS hospitals, as a tool to improve the health care offered to the Palestinian community living in Lebanon.

\section{Aim of the project}

The objective of the project, which was funded by the Italian Cooperation (Ministry of Foreign Affairs), was to endow the PRCS hospital network with a computerized case-mix hospital information system and develop the necessary skills for using it, in order to make data available to PRCS and UNRWA alike. The ground was prepared so that data could be used for: describing hospital activity and the health needs of Palestinian refugees; improving hospital management and evaluating health care costs; and assessing and improving the quality of care.

المجلة الصحية لشرق المتوسط، منظمة الصحة العالمية، المجلد الخنامس عشر، العدد ب، 9 +. 
The project was conducted by the PRCS and the Italian Cooperation, and was coordinated by Italian consultants together with resident Palestinian professionals, including a physician and an expert in information technology (IT). The project involved 2 phases: a preparatory phase (September 2002-June 2003), and an implementation and utilization phase (July 2003-October 2004). Currently registration of hospital information has become a routine activity, yet its utilization relies mainly on external personnel.

\section{Activities of the project}

\section{Preparatory phase}

During the preparatory phase the main activities were:

- to involve stakeholders in hospital information system programming;

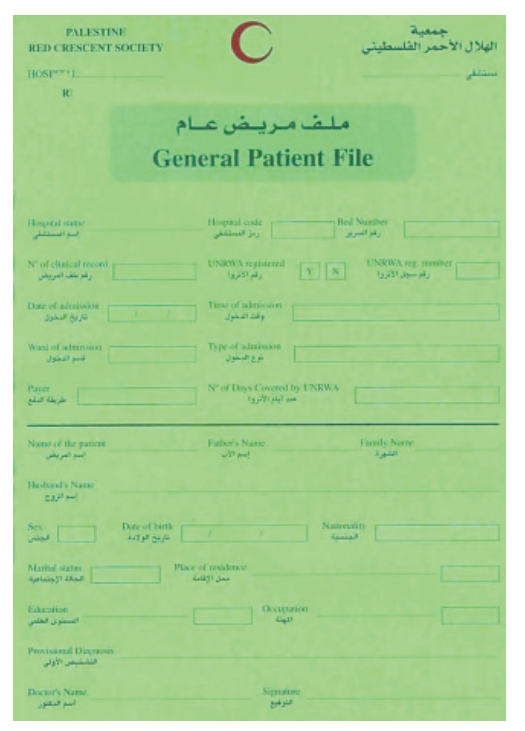

- to review the existing means of recording hospital data, identifying gaps and needs at different levels of the hospital network;

- to define the most appropriate reporting tools;

- to decide about the coding system for different types of information to be collected;

- to define methods for data collection and processing, and acquire the requisite materials; and

- to organize educational programmes for data managers and users.

After investigating the existing resources and reaching a consensus on the features and requirements of the new system, a discharge summary was identified as an adequate tool for registering information on each hospital episode. This was a paper form containing a minimum essential data set regarding the

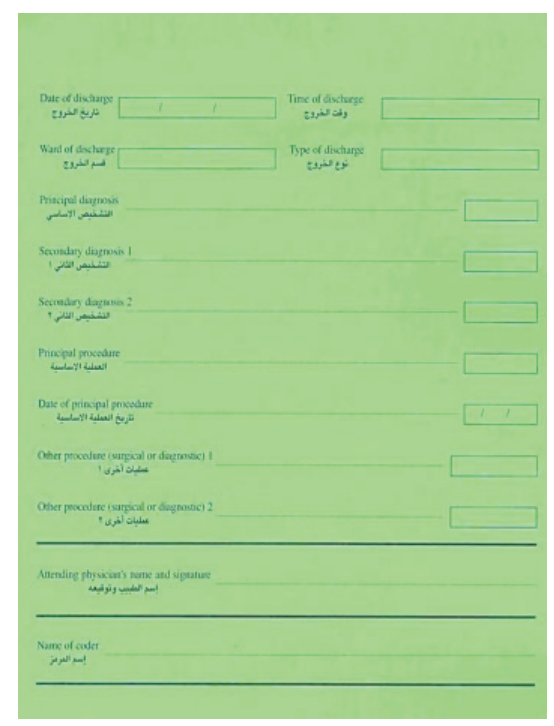


hospitalization episode [7] (Figure 1). The discharge summary is divided into $3 \mathrm{sec}-$ tions related to hospital administrative data, patient sociodemographic characteristics and clinical information.

All information is written in full and coded. Diagnoses are coded using the International Classification of Diseases and related health problems, 10th revision (ICD-10), and surgical and major medical procedures through the Current Procedural Terminology Codes (CPT4) issued by the American Medical Association. These coding systems have been adopted to align PRCS with the current Lebanese Ministry of Health Information System.

A Franco-Lebanese software engineering company was contracted to design the software database, to install it in all hospitals and to train PRCS personnel in using it. The software is a Microsoft Access database, simple to manage, user-friendly and compatible with most statistical analysis software or databases.

The training of PRCS staff at local universities was pivotal to implementing the information system. Administrative staff in charge of data entry were trained in computer and database use, selected medical staff received a 6-month training on coding clinical information and managing the information system and the PRCS project coordinators attended postgraduate training in data management and analysis for epidemiological and evaluative research.

All hospitals were equipped with a computer, printer, new clinical files and ICD-10 manuals.

The personnel in charge, identified among staff already employed by PRCS, carried out the new duties during working hours without any additional compensation, in order to streamline the project into ordinary tasks, and to set up the conditions for its sustainability at the end of the Italian support. The only incentive provided was in the form of training courses and educational materials.

\section{Utilization phase}

The second phase concentrated on:

- testing and using the system;

- developing data dissemination and feedback mechanisms; and

- summarizing the results and discussing them with the relevant stakeholders.

On 1 July 2003, all hospitals started filling in the discharge summary for every episode of discharge, and entering the information in the database. At admission of the patient, the staff member compiles the administrative and demographic sections of the discharge summary. At patient discharge, the trained medical staff member reports and codifies the diagnoses and procedures. Thereafter, the clinical files are returned to the administration office, where data are entered in the database at one time, within a few days after patient discharge.

Weekly visits by the IT supervisor aimed to ensure that all paper files were computerized in a timely manner and that errors and inconsistencies were corrected before the data were transferred electronically to the central computer at PRCS headquarters.

During the first 6 months of the new information system, the database was updated and modified numerous times to respond to unplanned requirements that arose from its utilization.

Data were analysed locally to produce reports for the medical directors, and transmitted monthly to PRCS headquarters in Beirut. The IT director, under the supervision of the medical coordinator, performed additional quality control at headquarters.

In June 2004, the system was running in all the PRCS hospitals and was able to

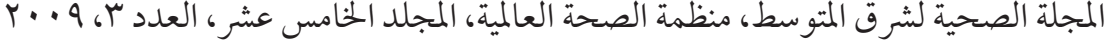


produce, as planned, routine information on PRCS hospitalization.

\section{Outcome of the project}

All project activities were completed in 2 years, relying on a local budget of about US\$ 100000 . The budget paid for training courses, local personnel and equipment, such as computers and software and educational materials $(42 \%, 26 \%$ and $32 \%$ respectively). Despite the limited resources available, the project succeeded in developing and completing a simple yet functional system, and hopefully in fostering PRCS self-trust, taking into consideration that most government-operated hospitals in Lebanon still do not have a computerized information system.

In October 2004, a 1-year PRCS hospital activity report was produced with the data collected, and presented at a seminar held at UNRWA headquarters in Beirut; the report is currently available online [8].

PRCS and UNRWA have also initiated talks to evaluate the possibility of basing reimbursement on the periodical release of data. This would make referral and reimbursement procedures more transparent, a goal repeatedly expressed in the proceedings of the conference on humanitarian needs of Palestine refugees held in Geneva in June 2004 [9] and in the URNWA medium-term plan 2005-09 [10] "to ensure that rational referral practices are adequately followed and implemented and that statistics on service utilization - actual durations of stay or types of procedures actually performedare not inflated or under-reported". It would also make available to URNWA information useful for epidemiological research: "improving UNRWA hospital management information systems and strengthening linkages with contracted hospitals in order to improve information exchange and feed- back on refugee patients treated in these hospitals".

In this light, holding the seminar at UNRWA headquarters in Beirut represented a positive step toward improving the synergy between the 2 organizations.

\section{Description of hospital activity and the} health needs of Palestinian refugees The system routinely generates individual data on all patients discharged from PRCS hospitals since July 2003. The analysis published in the above-mentioned report [8], although not the direct aim of this paper, shows the potential of the information system in describing the health services provided by the PRCS hospital network, both to external partners and to PRCS managers. More detailed analyses may address specific areas of care, in response to different information needs.

\section{Improving hospital management and evaluation of health care costs} Individual hospital directors have started producing reports and bringing them to the attention of the PRCS Scientific Committee regular meetings, to substantiate the requests related to resource and budget allocation. A good collaboration has emerged in some hospitals between clerks who manage the data and produce the reports and hospital directors who ask for specific information.

Moreover, following the implementation of the system, a project was financed by the Humanitarian Office of the European Commission and carried out by the Dutch Red Cross, with the aim of improving the financial sustainability of the PRCS hospital network through strengthening its hospital management. Three information systems have been created to audit the costs of hospital and ambulatory care, as well as the drug procurement cycle, and these have been based on the individual hospitaliza-

المجلة الصحية لشرق المتوسط، منظمة الصحة العالمية، المجلد الخنامس عشر، العدد ب، 9 +. 
tion records (except the one on ambulatory care). The project has been refinanced and is still running.

Assessing and improving quality of care The process of evaluating quality of care and developing good practice guidelines adapted to the local context, as already started in PRCS hospitals, has continued on request of PRCS with the methodological support of an Italian nongovernmental organization. The data collected by the hospital information system serve as baseline information for the selection of areas of care that need to be evaluated.

\section{Discussion}

We can consider the health context of Palestinian refugees in Lebanon as a microsystem where PRCS is one health care provider and UNRWA is the health agency or the commissioner; in such a scenario, the need for financial and performance accountability to ensure appropriate allocation of funds and quality of care becomes a priority [11]. Therefore, endowing the organization with such a tool enables it to describe and report the hospital services delivered, to evaluate the quantity and quality of care and to plan interventions to correct inefficiencies and minimize adverse outcomes.

\section{Epidemiological profiling and \\ comparability}

The information collected by the system is easy to retrieve, ready to analyse and, thanks to the use of internationally accepted diagnostic codes, offers the chance to compare PRCS hospital data with those collected elsewhere.

Such data allow analysis of the supply side, i.e. the "offer" of secondary care. If additional data on Palestinian hospitalizations reimbursed by UNRWA and treated by different providers in Lebanon are pooled with PRCS data, they can be used to estimate hospitalization rates and to describe the demand for secondary care among Palestinian refugees.

For those conditions for which hospitalization is a good proxy of disease-specific morbidity (e.g. hip fracture) they also allow epidemiological profiling of the hospitalized refugee population, and comparability among hospitals and geographic areas of residence.

\section{Fostering hospital management}

A basic hospital information system registering individual discharges represents a stepping-stone for the development of linked information systems, which together build up a management information system. Such a system allows cost analyses, quality standards protocols, patient referral systems, rationalization of expenses for drugs and acquisition of new technologies, and inventories of vehicles and equipment. Worldwide, information systems are thus becoming a core focus of hospital administration in district and regional health authorities.

In this framework, PRCS doctors and managers should continue to update and strengthen their capacity in terms of statistical analysis and epidemiology anmd health services research in order to exploit the full potential of the new system. PRCS staff participation in postgraduate and training courses together with their counterparts at Lebanese Ministry of Health level gave them a chance to feel part of the system, addressing their sense of exclusion and hopefully boosting their morale and self-trust.

Promote performance and financial accountability, and improve quality of care Health systems around the world face increasing pressures to provide health services effectively, efficiently and equitably.

المجلة الصحية لشرق المتوسط، منظمة الصحة العالمية، المجلد الخامس عشر، العدد ب، 9 +. 
Promoting performance accountability is often considered one way to improve quality of care, and reliable information systems are needed to measure service output and to design performance indicators and benchmarking comparisons among wards and hospitals [12].

Moreover, as hospital care is becoming a major budgetary item of total health expenditures in many countries, financial accountability acquires more relevance, particularly in the context of limited resources. Hospital information systems can also allow the shift to payment systems that maximize efficiency, quality of care, equity and consumer satisfaction [13], rather than per-day or fee-for-service payments, and eventually to remunerate hospitals according to case-mix.

The fact that PRCS is contracted by UNRWA is particularly relevant; their latest report in 2004 stressed that "morbidity statistics were collected for the first time in 2003 and their validity might be questioned because the hospital discharge notes are in many instances inadequate, which makes it difficult to classify morbidity conditions in the categories to which they belong" and concluded, among others, that "improving URNWA hospital management information systems and strengthening linkages with contracted hospitals in order to improve information exchange and feedback on refugee patients treated in these hospitals" is the future direction to follow [14].

The new information system is running in all PRCS hospitals and generates casemix information; having being operational only for a short time, it needs attentive maintenance and consolidation to continue to be reliable and fully exploited.

The new system represents a smart model of a fully computerized case-mix information system for the Lebanon health care sector. In the context of a refugee popu- lation living in disadvantaged conditions, such a system may foster PRCS self-trust and contribute to sustaining the social capital of the organization as well as supporting capacity building, and it represents a potential milestone for hospital management development [15].

However, in this phase the system is not yet fully integrated within the hospital management nor is it fully exploited by PRCS directors, and does not substitute for all registration forms.

\section{Lessons learned}

We believe that the effort in training health managers and medical directors to make use of the data should go in parallel with the implementation of the information system. The main risk is to keep in place an underused system which constitutes a burden for data-entry staff and managers, with no benefits for the organization and that produces poor quality data.

The mutual and self-reinforcing relationship between quality of data and its utilization is well acknowledged. It is mandatory that data be used at all levels, from hospital wards and departments to medical directors at headquarters, to satisfy information needs and to promote clinical governance. It is also strategic for supporting capacitybuilding that information is shared among all stakeholders, and that indicators of activity are fed back to data managers, if the value and potential of these are to be fully appreciated and exploited.

Moreover, once the system is routinely used, updates will be required by hospital managers, as happened during its development; therefore the system needs continuous maintenance and attention.

Finally, we believe that the flexibility and essential features of the system allowed it to be adapted to the new information need when the Dutch Red Cross programme was 
initiated, in addition to guaranteeing its sustainability.

\section{Future directions}

Other aid projects have been recently be funded to support PRCS to focus on the implementation of different information systems (centred on the individual patient file) to document specific aspects of PRCS hospital activity; others are concentrating on improving areas of hospital care identified through the analysis of data generated by the system. This may suggest that the intrinsic value of the new system has been recognized and is making a positive impact.
Though the achievements of the project over 2 years are encouraging, the analysis and use of the data collected is not yet routine. External partners or ad hoc experts must continue their technical support to PRCS to ensure a continuous strengthening of the system and its streamlining into current activities, until a culture of information is created at the managerial level.

\section{Acknowledgements}

We would like to thank Elham Yaacoub and Dr Salah Al Ahmad at PRCS for their continuous support and collaboration in all project activities in Lebanon.

\section{References}

1. Report of the Commissioner-General of the United Nations Relief and Works Agency for Palestine Refugees in the Near East. General Assembly official records, sixtieth session, Suppl. No. 13. New York, United Nations, 2005 (A/60/13).

2. Madi $\mathrm{HH}$. Infant and child mortality rates among Palestinian refugee populations. Lancet, 2000, 356:312.

3. Lebanon: economic and social rights of Palestinian refugees: submission to the Committee on the Elimination of Racial Discrimination. Lebanon, Amnesty International, 2003 (MDE 18/017/2003).

4. Abubaker W, Abdulrahman M. Quality assurance guides health reform in Jordan. Q.A. brief, 1996, 5:19-21.

5. Rawabdeh AA. Health care cost containment strategies: the Jordanian experience. International journal of health planning and management, 2005, 20:53-66.

6. Van Lerberghe $W$ et al. Reform follows failure: I. Unregulated private care in Lebanon. Health policy and planning, 1997, 12:296-311.
7. Developing health management information systems. A practical guide for developing countries. Manila, World Health Organization Regional Office for the Western Pacific, 2004.

8. Othman $\mathrm{M}$ et al. The new PRCS hospital information system in Lebanon. Report of one-year hospital activity. Beirut, Italian Cooperation, 2004 (http://www.asplazio.it/asp_online/att_ospedaliera/files/ files_valutazione_esterna/san_internaz/ rapporto libano.pdf, accessed 17 November 2008)).

9. Conference on humanitarian needs of Palestine refugees convenes at Geneva. In: Committee on the Exercise of the Inalienable Rights of the Palestinian People (CEIRPP). Bulletin on action by the United Nations system and intergovernmental organizations relevant to the question of Palestine. May-October 2004, Volume XXVII, Bulletin No. 2 (http://domino. un.org/unispal.nsf/bb6fe1e728031318 $85256 \mathrm{c} 380071 \mathrm{~d} 04 \mathrm{c} / 02855$ ed4ed05a4 dc85256fe30056930b!OpenDocument, accessed 16 January 2009).

المجلة الصحية لشرق المتوسط، منظمة الصحة العالمية، المجلد الخامس عشر، العدد با، 9 . · 
10. UNRWA: medium term plan (MTP) 20052009. A better future for Palestinian refugees. Gaza, United Nations Relief and Work Agency for Palestine Refugees in the Near East, 2005 (http://www.un.org/unrwa/ news/mtp.pdf, accessed 16 January 2009).

11. Abramson WB. Monitoring and evaluation of contracts for health service delivery in Costa Rica. Health policy and planning, 2001, 16:404-11.

12. Brinkerhoff DW. Accountability and health systems: toward conceptual clarity and policy relevance. Health policy and planning, 2004, 19:371-9.

13. Roger France $\mathrm{FH}$ et al. Case mix: global views, local actions. Evolution in twenty countries. Amsterdam, IOS Press, 2001 (Studies in Health Technology and Informatics, Vol. 86).

14. Review of hospital services (policies, utilization trends and cost-analysis). Gaza, Health Department, United Nations Relief and Work Agency for Palestine Refugees in the Near East, 2004.

15. Shaw C. How can hospital performance be measured and monitored? Copenhagen, WHO Regional Office for Europe, 2003 (Health Evidence Network report) (http://www.euro.who.int/document/ e82975.pdf, accessed 6 August 2008).

\section{Management Effectiveness Programme}

The Management Effectiveness Programme (MEP) is an evolving system of management practices, tools, roles and training methods adapted from various fields for creating quality health systems to improve the health of the population. The MEP is intended to be an integral part of the national health systems development and improvement efforts of the country. It focuses on the development of selected sites, which could be a health district, hospital, laboratory, health departments at national or peripheral levels, etc.

The MEP supports site health leaders and their teams in identifying weaknesses in their system and developing their capacity to improve their management processes and practices. The MEP is designed to integrate the fundamental principles of quality management into the management practices and processes of the national health system. The main strategy for implementing the MEP combines a learning technology in the form of core modules with a systems and roles development approach for each site. Further information on MEP is available at: http://www.emro.who.int/mei/mep/ 